
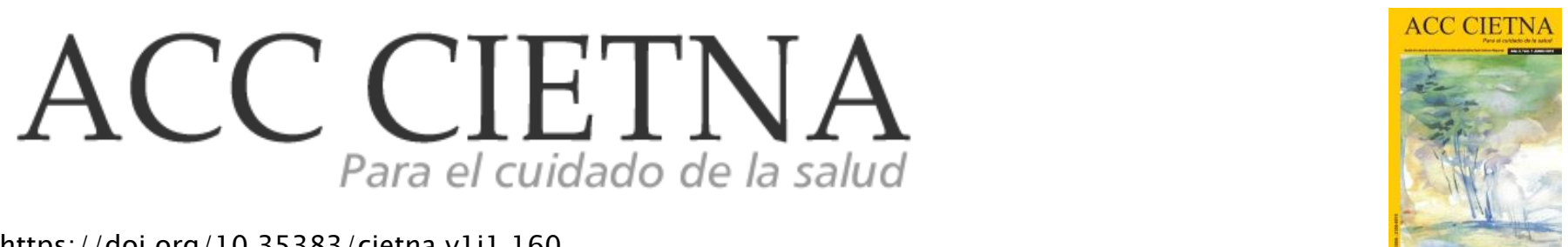

https://doi.org/10.35383/cietna.v1i1.160

\title{
Adaptación al duelo anticipado de padres de escolares con Leucemia Linfoblástica Aguda. Hospital Nacional Almanzor Aguinaga Asenjo
}

\author{
Sánchez Vásquez Tatiana ${ }^{1}$, Llano García Dina ${ }^{2}$, Guerrero Quiroz Elizabeth Soledad ${ }^{3}$
}

\begin{tabular}{l} 
INFORMACIÓN DEL ARTÍCULO \\
\hline Historia del artículo: \\
Recibido el 23 de junio de 2013 \\
Aceptado el 30 de noviembre de 2013
\end{tabular}

Palabras claves:

Duelo anticipado

Leucemia fibroblástica aguda

Padres de escolares.

\section{RESUMEN}

La presente investigación se desarrolló en una institución hospitalaria de IV nivel del EsSALUD, durante el 2010, con el objetivo de describir y comprender el duelo anticipado de los padres de escolares con leucemia Linfoblástica Aguda. La construcción del marco teórico conceptual se fundamenta con la teoría de Callista Roy, para adaptación; y en Tizón García, para duelo anticipado. Esta investigación es de carácter cualitativo con abordaje de estudio de caso, tuvo como sujetos de investigación 08 padres de familia que se encontraban las 24 horas del día al cuidado sus hijos que padecían de leucemia linfoblástica aguda. Se tuvo como escenario el servicio de hematología - oncología del Hospital Nacional Almanzor Aguinaga Asenjo (HNAAA). La técnica de recolección de datos que se utilizó fue la entrevista abierta semi estructurada. Durante la investigación se respetaron los principios éticos y de cientificidad y se firmó el consentimiento informado. Producto del análisis temático, surgieron cuatro categorías, donde los padres manifestaron haber experimentado sentimientos de dolor, como: tristeza y preocupación; y sentimientos de culpa que generaron en ellas reacciones de negación y búsqueda desesperada de soluciones que salven la vida de sus hijos. Situación que las llevó a buscar apoyo en Dios, en su familia y también en el profesional de enfermería que está al cuidado de sus niños.

\footnotetext{
1 Licenciada en enfemeria. Enfermera del Centro de salud Zaña, Chiclayo, Perú. Email: tsanchez@gmail.com

2 Licenciado en enfermería. Enfermera del Hospital Metropolitano, Chiclayo, Perú. Email: dllano@hotmail.com

${ }^{3}$ Enfermera especialista en Neonatología del Hospital Nacional Almanzor Aguinaga Asenjo y profesora adjunta a la Escuela de Enfermería de la 
Adaptation to the anticipated grief of parents of children with acute lymphoblastic leukemia. National Hospital Almanzor Aguinaga Asenjo

\section{ABSTRACT}

Keywords:

Duel

Acute lymphoblastic leukemia Parents
This research was carried out at EsSalud hospital institution of fourth level, during the year 2000, its aim is describe and understand duel of school father with acute lymphoblastic leukemia.The conceptual theorical framwork is founded by Callista Roy theory, for adaptation, and Garcia Tizon for anticipatory duel .This research is qualitative with case study approach, as research subjects had eight parents who were 24 hours in the care of their children suffering from acute lymphoblastic leukemia.It took place at the service of the national hospital Almanzar Aguinaga Asenjo. The Data collection technique that was used was an semi structured interview open. During the investigation, has been respecting the ethical principles and scientificity and signed informed consent. thematic analysis product, emerged four categories, where parents said they had experienced feelings of pain, such as sadness and concern and guilt, which generated in reactions of denial and desperate search for solutions that save the lives of their children. Situation that had led them to seek support from God, their family and also staff nursing who are caring for their children such as sadness and concern and guilt, which generated in reactions of denial and desperate search for solutions that save the lives of their children.

\section{Introducción}

En la actualidad el cáncer es la segunda causa de muerte en el niño, después de los cinco años de edad. Las estadísticas demuestran que cada año más de 160000 niños son diagnosticados con cáncer en el mundo. El $80 \%$ de los pacientes infantiles viven en países en desarrollo. Según la Unión Internacional Contra el Cáncer (UICC), en los países desarrollados, 3 de cada 4 niños con cáncer sobreviven al menos cinco años después de ser diagnosticados, gracias a los progres os en el diagnóstico y tratamiento de esta enfermedad. En Los países en desarrollo, más de la mitad de los niños diagnosticados con cáncer tiene probabilidades de morir. 1

El padecer de cáncer infantil produce un impacto psicológico muy fuerte en la familia, donde todos sus miembros se ven afectados emocionalmente por la enfermedad, cuando los padres reciben la noticia de que un hijo tiene leucemia o linfoma, se enfrenta a la inseguridad de saber la probabilidad de vida de su hijo.

Es un tiempo lleno de nuevas personas y situaciones, preocupaciones y cambios, puede provocar una mezcla de reacciones, que incluyen shock y confusión, negación, miedo, ansiedad, enojo, dolor y tristeza. Muchos padres sufren esta mezcla de sentimientos durante toda la enfermedad de su hijo, hasta lograr el proceso de adaptación. Todas estas reacciones son válidas ante su situación. Algunos padres experimentan un "duelo anticipado", en donde se produce una reacción de angustia desencadenada por la situación límite que están pasando, por el futuro incierto y las expectativas dolorosas avizorando un desenlace fatal. ${ }^{2}$ 
Con respecto al proceso en el que entra, tanto el sistema familiar, como de manera personal cada uno de sus miembros, al recibir el diagnóstico, aparece de inmediato el miedo a la pérdida que deben comenzar a plantearse, y surge aunque de distinta manera, el proceso de duelo, el cual ha sido identificado como un proceso que consta de varias fases o etapas, el cual los padres de familia tienen que adaptarse y posteriormente resignarse al desenlace final'2.

Las investigadoras durante sus prácticas hospitalarias en el Hospital Nacional Almanzor Aguinaga Asenjo, especialmente en el servicio de Oncología, pudieron observar la constante lucha de los padres de escolares con Leucemia linfoblástica aguda, por lograr adaptarse al duelo anticipado que están atravesando y a los cambios que les toca vivir, ya que ellos provienen de sectores apartados del Hospital, por lo que la distancia y el costo económico que implica el traslado de ciudad, genera un gran esfuerzo para llegar al centro asistencial, lo que provoca en las amilias una separación temporal, ya que son los padres o uno de ellos, los que acompañan al niño, teniendo que hospedarse en casas de acogida o en hogares de ayuda al niño con leucemia.

Ante la realidad encontrada las investigadoras formularon el siguiente problema científico ¿Cómo es el proceso de adaptación al duelo anticipado de los padres de escolares con leucemia linfoblástica aguda?

La investigación tuvo como objetivo, describir y comprender la adaptación al duelo anticipado de los padres de escolares con leucemia linfoblástica aguda, ya que el sistema cambia, y lo hace de manera brusca, cada uno de los miembros sufre; primero, ante la situación de la enfermedad y segundo, ante los drásticos cambios que esta trae consigo. Los padres, quienes son pilares fundamentales dentro de todo sistema familiar de un instante a otro, se ven susceptibles $y$ vulnerables al asumir el nuevo acontecimiento, tienen presiones económicas, laborales, familiares y sociales; la familia entra en crisis, su equilibrio se ve intervenido, su dinámica cambia y su funcionamiento familiar adquiere un nuevo sentido ${ }^{3}$.

Esta investigación es relevante, puesto que contribuye a conocer en forma más profunda el proceso de adaptación al duelo anticipado de los padres tras el diagnóstico de leucemia linfoblástica aguda, de manera que sea una herramienta de trabajo con la familia del niño hospitalizado y el equipo de salud. Además, podrá contribuir a que todas las enfermeras que trabajen con niños que padecen de esta enfermedad, el cuidado centrado en la familia para su atención; la capacidad y sensibilidad para comunicarse de forma asertiva con los niños y sus familiares, ya que los padres pasan por un proceso de adaptación que un inicio es muy difícil y necesitan todo el apoyo moral.

\section{Metodología}

Es una investigación cualitativa enfocada en el estudio de caso, diseño que estudia un fenómeno contemporáneo dentro de su contexto de la vida real, la principal ventaja es la profundidad a la que se puede llegar cuando se investiga un número reducido de individuos, instituciones o grupos ${ }^{4}$.

La investigación también se desarrolló tomando en cuenta los momentos descritos por Nisbet y Watt, citado por Pineda 5 , quienes caracterizan el desarrollo del estudio de caso en tres fases: Exploratoria, la que se inició con la observación de una realidad concreta, existieron algunas preguntas o puntos críticos que fueron explicados, reformulados o eliminados a medida que se mostraron más o menos relevantes en la situación estudiada. Se identificó la problematización, para la cual se plantearon preguntas orientadoras al inicio, esta fase fue fundamental para la definición más precisa del objeto de estudio, puesto que en esta fase se definió el escenario; y a la vez se estableció el contacto con los sujetos de estudio. 
Asimismo, en la segunda fase denominada delimitación del estudio, las investigadoras procedieron a recolectar la información de forma sistemática, utilizando diferentes instrumentos o técnicas seleccionados por las características propias del objeto de estudio para ello la entrevista abierta a profundidad. Esta fase se ejecutó en el servicio de hematología - oncología del Hospital Nacional Almanzor Aguinaga Asenjo, en el cual se captó al profesional de enfermería que trabaja en esta área; quienes fueron el nexo principal para realizar la entrevista a 8 padres de familia que se encontraron en dicho servicio, con la finalidad de validar y corroborar la información. Para ello, se empleó como instrumento de recolección de datos, la entrevista semiestructurada.

Finalmente, en la tercera fase denominada, análisis sistemático y elaboración del informe, tuvo como principal tarea que las investigadoras realizaran la revisión de las entrevistas para procesar la información, analizarla y tornarla disponible a los informantes, de forma que estos pudieran manifestar sus reacciones sobre el significado, tomando en cuenta los objetivos de la investigación para luego obtener categorías que contribuyan en la construcción del caso en estudio.

Los sujetos de la investigación fueron 8 padres de familia, con hijos en edad escolar, con diagnóstico de Leucemia linfoblástica aguda.

Asímismo, siguiendo el principio ético de confiabilidad, las identidades de los sujetos de estudio se protegieron mediante el seudónimo de nombres de rosas y sus discursos fueron presentados a lo largo de la investigación.

El análisis temático de esta investigación es de carácter cualitativo, y procura lograr la descripción holística, esto quiere decir que intenta analizar exhaustivamente, con sumo detalle, un asunto o actividad en particular, se realiza a grupos pequeños, donde se permite trabajar con datos subjetivos, como experiencias, actitudes, creencias, pensamientos y reflexiones. Además, pretende comprender a través de la observación e investigación la realidad de un fenómeno que los incluye, efectuado a través del análisis de documentos, tales como la realización de entrevistas y aplicación de cuestionarios, seguido de observaciones sistemáticas realizadas por las investigadoras.

La presente investigación se respaldó en los principios éticos personalistas de Elio Sgreccia ${ }^{6}$, él propone cuatro principios que operan como ayuda y guías de la acción en la consideración de cada decisión relativa a la investigación con seres humanos, de los cuales se mencionarán tres que se ajustan a la investigación: El principio de inviolabilidad de la vida humana, la libertad y responsabilidad, sociabilidad y subsidiariedad.

La calidad de una investigación científica se determina a través del rigor metodológico con el que ha sido señalada y desarrollada la veracidad de los resultados, teniendo en cuenta la consideración de los principios propuestos por Lincol y Guba se tuvo en cuenta los criterios de credibilidad, confiabilidad y validez, transferibilidad.

\section{Análisis y discusión}

\section{Negando el diagnóstico médico:}

La mayoría de los padres desearían creer que el diagnóstico de cáncer de sus hijos es un error, este se convierte en uno de los primeros mecanismos que permite al individuo rehusarse a admitir que existe un problema de salud en los niños, de tal manera que utilizan estos mecanismos para rechazar los sentimientos dolorosos que conlleva la enfermedad, negándose rotundamente aceptarla.

Según Midlenton7, “los padres jamás están preparados para escuchar que su hijo tiene una enfermedad que amenaza su vida. Al principio, dependiendo del conocimiento o experiencia que tengan acerca del cáncer, ellos pueden pensar que el diagnóstico es una sentencia de muerte. Sienten 
que su hijo va a sufrir y que su familia experimentará una larga agonía. A menudo describen una sensación de aturdimiento como si hubieran recibido un fuerte impacto físico. Dicen sentirse confundidos o incapaces de oír, recordar o pensar con claridad cuando reciben información acerca del diagnóstico o plan de tratamiento de su hijo. Esta categoría se refleja en las siguientes afirmaciones mencionadas por los padres:

\section{"Señorita me negaba rotundamente al diagnóstico} que me dieron sobre la enfermedad de mi niño, fue un noticia inesperada para todos en casa, no aceptábamos la realidad, pensábamos que todo un sueño que estábamos viviendo" (Margarita).

\section{Dejando todo por cuidarlo: Modificando mi ritmo de vida.}

Tener un niño enfermo interrumpe el normal funcionamiento en la familia, ya que uno de los padres es el encargado del cuidado permanente durante la estancia hospitalaria, es por ello que uno de los cónyuges tiende abandonar sus obligaciones laborales, para dedicarse únicamente al cuidado del hijo enfermo, dando como consecuencia la modificación en el ritmo de vida de esta persona.

Según Soler 8 , los padres que son el pilar dentro del hogar, muchas veces tienen que abandonar sus labores cotidianas, para ocuparse de su hijo enfermo, cambiando así su ritmo de vida. Desligándose el siguiente discurso:

"Tuve que dejar a toda mi familia para poder cuidar a mi hijo que está acá en el hospital, dejé todo, mi trabajo, y mis demás responsabilidades que antes tenía, esta enfermedad hace cambiar nuestras vidas, para dedicarse de lleno al cuidado del niño enfermo" (Clavel).

\section{Tristeza vs/ Resignación a la enfermedad y muerte:}

Enfrentarse a un diagnóstico de cáncer en un hijo es una experiencia muy estresante y dolorosa. Una de las reacciones emocionales más frecuentes es la tristeza, ya que esta enfermedad se presenta como una pesadilla para los padres. Siendo una reacción común y apropiada ante el diagnóstico una enfermedad terminal que puede transcurrir en el tiempo.

Para Lorraine ${ }^{9}$ la tristeza es una experiencia que presentan una serie de reacciones emocionales más frecuentes, siendo esta una reacción fácilmente identificable, comunicable y reconocible, tanto por los padres como por la persona que padece la enfermedad. Los padres se sienten tristes, apenados por los procedimientos médicos utilizados y tratamientos que reciben sus hijos y la ansiedad que genera en ellos. Suele ser peor para los niños, pero también para los padres que frecuentemente se sienten angustiados e impotentes ante tal situación. La mayoría de los padres tratan de sobrellevar la enfermedad mostrando sentimientos diferentes delante de ellos, procurando mostrar tranquilidad, ser fuertes, no flaquear en la presencia de ellos, aunque por dentro sienten una sensación de pérdida, una experiencia muy abrumante, que se refleja en sentimientos de tristeza, impotencia y rechazo ante la enfermedad ${ }^{10}$.

"Es triste enterarse de una enfermedad tan grave que es la leucemia, esta nos afecta emocionalmente a toda la familia, ya que nos sentimos impotentes de no poder resolver el problema de mi niño" (Amapola).

\section{Mi Dios nunca me abandona: Buscando apoyo en un Ser Superior:}

La enfermedad de un hijo para los padres puede parecerles injusta. Esta sensación puede Ilevarlos a cuestionar, desde su punto de vista, el significado de tener un niño sano y enfermo, ven de otra manera el valor de la vida y la relación con Dios. Piensan que a veces es un castigo, por todas los pecados cometidos, aunque muchas veces estos padres suelen aferrarse a un Ser superior, se entregan de todo corazón al Omnipotente 
teniendo la esperanza que él lo va a sanar. Para muchos padres resulta útil la exploración de estos sentimientos con la ayuda de un consejero o asesor espiritual que le ayuda a superar día a día la enfermedad de su niño ${ }^{11}$.

Middleton 7 menciona que, en muchos casos, por la desesperación y el miedo, las personas se aferran a un Ser Supremo, porque es el único que puede salvar a su hijo. Las creencias espirituales y religiosas de un Ser Supremo es importante en la vida de estos padres ya que influye en sus estilos de vida, actitudes y sentimientos relativos con referente a su enfermedad, pudiéndola ver como una prueba de fe, aferrándose y confiando más Dios, en la Virgen María, siendo ellos su única fortaleza para llevar la enfermedad de su hijo. Para estos padres la enfermedad, con mucha frecuencia, empuja a una búsqueda incansable de Dios, refugiándose y aferrándose a Él, es su estrategia factible, que ellos han desarrollado para llenarse de fortaleza, serenidad, y para hacer frente a una nueva situación como es la enfermedad de su hijo. Apreciemos los siguientes discursos:

"Señorita, ahora mi único consuelo es mi Dios, sé que él nunca nos va abandonar, siempre le rezo, participo de misa todos los domingos encomendando la enfermedad de mi niño para que se recupere pronto" (Margarita).

En los párrafos anteriores podemos observar el gran impacto que produce el apoyo en un Ser Supremo dentro de la familia con un hijo que tiene cáncer, las personas se aferran a la fe que los hace más fuertes, sienten que Dios nunca los van a abandonar y que él les hará el milagro de curar a su hijo de la enfermedad. Así logran tener mayor fortaleza para superar los inconvenientes de la enfermedad, ya que esta crea situaciones de estrés, por sentimientos de amenaza y pérdida, que dificultan la activación y puesta en marcha de estrategias y mecanismos interiores para afrontarla, pero es obvia la necesidad de que las personas aprendan el conocimiento espiritual, de modo que sean capaces de afrontar dignamente la enfermedad de sus hijos.

\section{Consideraciones finales}

- El diagnóstico de cáncer infantil afecta emocionalmente a toda la familia, especialmente a los padres, causa incertidumbre, preocupación, sentimientos de culpa que generarán en ellos reacciones de negación y búsqueda desesperada de soluciones que salven la vida de sus hijos.

- El diagnóstico de cáncer en un hijo interrumpe el normal funcionamiento en la familia, ya que uno de los padres es el encargado del cuidado permanente durante la estancia hospitalaria, por lo que muchas veces se ve obligado abandonar las labores que tiene que desempeñar, para dedicarse únicamente al cuidado del hijo enfermo, dando como consecuencia la modificación en el ritmo de vida.

- Las experiencias son muy abundantes y dependen de las circunstancias específicas de cada familia, existiendo en los padres sentimientos comunes de tristeza, resignación, impotencia y rechazo de la enfermedad y al tratamiento. En los primeros momentos existe una gran angustia $y$ tristeza ante la amenaza de la muerte que perciben, sentimientos que van cambiando según avanza el tiempo y comprueban que su hijo sigue vivo y que va superando el tratamiento, llegando a resignarse ante la enfermedad de su niño que la padece.

- La fortaleza principal de los padres de los escolares con Leucemia linfoblástica aguda fue buscar apoyo en Dios, en su familia y también en el profesional de enfermería que está al cuidado de sus niños, el que según sus percepciones brinda un buen cuidado asistencial, pero descuida la parte humana fundamental en el cuidado holístico e integral que debe brindar, evidenciando que persiste el paradigma biomédico tradicional en el cuidado de enfermería. 


\section{Bibliografía}

1. INEI. $\quad 1999 . \quad$ Disponible: htth/www.inei.gob.pe/biblioinei.Ipub/bancopub/ est/liboo 78/so4-4. Htm. Consultado el 2010 setiembre 16

2. Lagos, E y otros. Estrategias de afrontamiento en padres de niños con cáncer.2004. Disponible en www.redalyc.uaemex.mx/redalyc/pdf/264/26413 205.pdf. Consultado el 2011 febrero 02.

3. Dossier, B. Fundamentos de enfermería: conceptos, proceso y práctica. $5^{\circ}$ ed. Madrid: Mc Graw-Hill Interamericana; 1999.

4. Bernal, C. Metodología de la investigación. $2^{\circ}$ ed. México: Pearson Educación; 2006.

5. Pineda, Beatriz Elia y otros. Metodología de la investigación: Manual para el desarrollo de personal de salud. $2^{\mathrm{a}}$ ed. Washington: Organización Panamericana de la Salud; 1994.
6. Sgreccia, E. Bioética personalista: Principios y orientaciones. I Congreso Internacional de Bioética Universidad Católica Santo Toribio de Mogrovejo. Chiclayo-Perú, 14-16 de octubre de 2008.

7. Midleton, J. Yo no quiero tener cáncer. Ed. Grijalbo. Santiago, Chile

8. Soler, $\mathrm{M}^{\mathrm{a}}$.1996. Afrontamiento y adaptación emocional en padres de niños con cáncer. Disponible en la web: www.geocities.com/CollegePark/Library/7893/IV b.htm. Consultado el 2011 noviembre 09.

9. Lorraine, Shey. Agonía, muerte y duelo. Ed. Manual Moderno. México. 2005.

10. Rojas, E. "Alegría, tristeza y resignación". 1994. (Documento en línea).http://www.enriquerojas.com/ficha_articulo .asp?ld=42>.Consultado el 2011 mayo 12.

11. Sánchez, P. "La fe en Dios". 2006. Disponible en:http://www.escuelabiblicas.org/material/12 La FeEnDios.pdf.Consulta Mayo del 2011 